\title{
Phototermal self-excitation of nanomechanical resonators in liquids
}

\author{
D. Ramos, J. Mertens, M. Calleja, and J. Tamayo ${ }^{\text {a) }}$ \\ BioNanoMechanics Lab, National Centre for Microelectronics, IMM-CNM, CSIC Isaac Newton 8 (PTM), \\ Tres Cantos 28760, Madrid, Spain
}

(Received 26 March 2008; accepted 9 April 2008; published online 30 April 2008)

\begin{abstract}
We report the use of the photothermal actuation for the self-excitation of a selected vibration mode of a microcantilever in liquid. The gain of the positive feedback loop is adjusted in order to obtain a negative effective damping. In this regime, the amplitude noise is squeezed due to the nonlinear saturation of the system and the phase noise is largely reduced. The microcantilever vibration achieved a frequency stability of the order of $1 \mathrm{ppm}$ for a bandwidth of $1 \mathrm{~Hz}$. This is at least two orders of magnitude better than previous measurements in liquids. The obtained sensitivity is applied for detecting in real time the change of the fluid properties when glycerol is added to water at a concentration of 1\% ( $\mathrm{m} / \mathrm{m})$. (C) 2008 American Institute of Physics. [DOI: 10.1063/1.2917718]
\end{abstract}

The nanometer-scale vibration of flexible microstructures such as microcantilevers can be exquisitely tuned by a number of interactions. Temperature, electrical and magnetic fields, intermolecular forces, molecular adsorption, and fluid properties (density and viscosity) can sensitively shift the natural vibration frequencies. This intimate connection between a nanomechanical resonator and its surrounding is the basis of scanning probe microscopes ${ }^{1}$ and recently developed nanomechanical sensors for chemical and biological detection and for measurement of rheological properties of fluids. ${ }^{2}$ The performance and sensitivity of these applications depends on the rate of energy dissipated by the resonator. The energy dissipation is measured by means of the quality factor $(Q)$ that can be written as $Q=2 \pi W_{0} / W_{\text {dis }}$, where $W_{0}$ and $W_{\text {dis }}$ are the stored vibrational energy and the energy lost per oscillation cycle, respectively. ${ }^{3}$ The connection between dissipation and sensitivity is given by the fact that $Q$ can also be written as $Q \approx 3^{1 / 2} \omega_{0} / \Delta \omega_{\text {fwhm }}$, where $\omega_{0}$ is the angular resonant frequency and $\Delta \omega_{\mathrm{fwhm}}$ is the full width at half maximum of the resonance peak. ${ }^{4}$ For microcantilevers vibrating in fluids, the major source of dissipation is the viscous damping, and this explain why the best performance of scanning probe microscopes and nanomechanical sensors have been attained in vacuum ${ }^{5,6}$ where $Q \approx 10^{3}-10^{4}$. The $Q$ decreases in air $^{7,8}$ to 20-500 and in liquids ${ }^{9,10}$ to $1-5$. The low $Q$ in liquids severely degrades the sensitivity of nanomechanical resonators to obtain major structural and functional information of biological systems and for the development of sensitive biosensors and rheometers working in real time.

Here, we propose a technique for measuring the resonant frequency of nanomechanical resonators in liquid based on (i) photothermal actuation for efficient and selective excitation of flexural vibration modes of microcantilevers ${ }^{11,12}$ and (ii) the self-excitation of the cantilever by a positive feedback loop in the saturation regime. ${ }^{13,14}$ We achieve a frequency resolution of the order of $1 \mathrm{ppm}$. The potential of the technique is demonstrated by measuring in real time the effect of adding $1 \%$ of glycerol in water.

The experiments were performed with silicon nitride microcantilevers $200 \mu \mathrm{m}$ long, $20 \mu \mathrm{m}$ wide, and $0.8 \mu \mathrm{m}$ thick. For the photothermal actuation, the cantilevers were coated

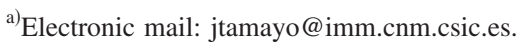

with a $70 \mathrm{~nm}$ thick gold film by e-beam evaporation. The cantilevers were placed in a homemade fluid cell with an inlet and an outlet ports for fluid exchange. The cantilever vibration was detected by the optical beam deflection method by a green laser diode $(532 \mathrm{~nm}, 5 \mathrm{~mW}$, Edmund Optics, Ltd.) and a quadrant photodetector (Hamamatsu Photonics). The photothermal excitation of the cantilevers was achieved by a red laser diode beam whose intensity can be modulated from dc up to $500 \mathrm{kHz}(5 \mathrm{~mW}, 635 \mathrm{~nm}$; Edmund Optics, Ltd.). The self-excitation was performed by using commercial electronics (Infinitesima) and a combination of current and voltage amplifiers.

An optical micrograph of a cantilever illuminated by the sensing (green) and excitation (red) laser beams is shown in Fig. 1. Both beams are strongly focused producing a spot size on the cantilever of about $10 \mu \mathrm{m}$. The intensitymodulated laser induces a time-periodic temperature change around the illuminated cantilever region, which gives a cantilever vibration via the bimorph effect. The optimal excitation of a flexural vibration mode is achieved by focusing the intensity-modulated laser beam close to the regions of maxima curvature of the vibration shape. ${ }^{11}$ Here, the second vibration mode was selected by focusing the intensitymodulated laser beam near the antinode position at about $110 \mu \mathrm{m}$ from the base. The resonant frequency was obtained by two methods. In the first method, the driving signal was frequency modulated (FM) in synchronization with the acquisition time. Hence, frequency spectra can be obtained in a few tens of millisecond. The second method is the selfexcitation technique presented here. The output of the position-sensitive photodetector is filtered by a high-pass filter with a cutoff frequency of $10 \mathrm{kHz}$, amplified by a variable gain amplifier, shifted by a variable phase shifter and then connected to the input of the excitation laser. ${ }^{4,13}$ The phase shift was approximately adjusted to $\pi / 2 \mathrm{rad}$.

Figure 2(a) plots the frequency spectra of the cantilever in water; without excitation, driven by the FM signal and self-driven by the positive feedback loop. Without excitation, the graph clearly shows the resonance peak of the second flexural vibration mode at $39.4 \mathrm{kHz}$ with $Q=2.5$. This implies that the Brownian motion of the cantilever is well above the electronic noise in this frequency range. The FM driving signal reproduces the resonance peak with higher signal-to-noise ratio, but without any change in the fre- 

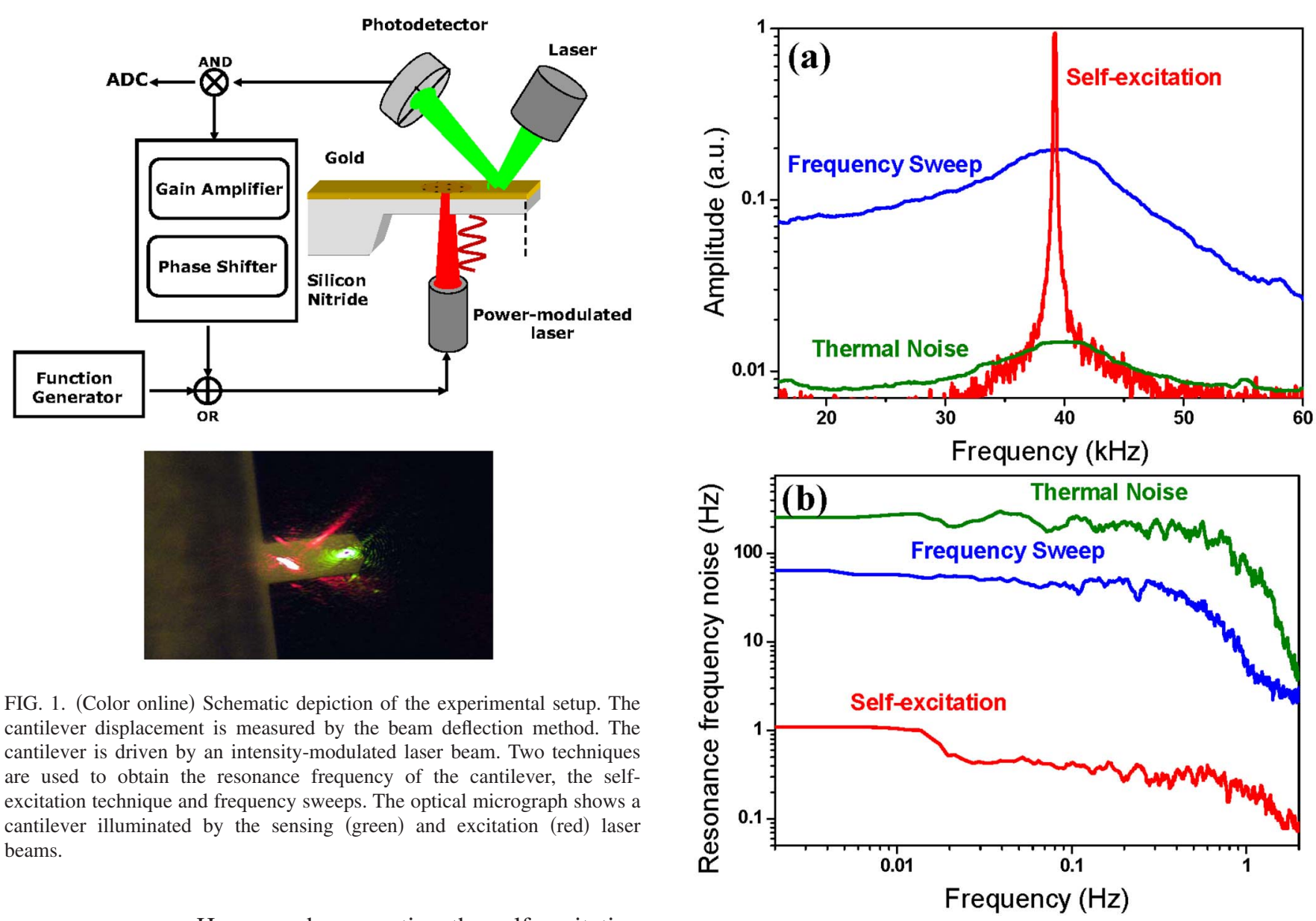

quency response. However, by operating the self-excitation technique in the nonlinear regime, a very narrow resonance peak with a linewidth of $180 \mathrm{~Hz}$ was achieved. Equally important, the peak preserved its amplitude and linewidth for several hours. Self-excitation in the linear regime ${ }^{4}$ leads to an effective enhancement of the quality factor $\left(Q_{\text {eff }}\right)$ by $Q_{\text {eff }}^{-1}=Q^{-1}-G / k$, where $k$ is the spring constant and $G$ is the gain in the feedback loop that relates the excitation force to the cantilever vibration. Although an effective enhancement of $Q$ of about two orders of magnitude can be achieved, the extremely sharp dependence of the oscillation on the gain leads to strong fluctuations in the amplitude and frequency. By adjusting the gain to values just above the critical gain $G_{c}=k / Q$, the oscillation enters on the nonlinear regime. ${ }^{13} \mathrm{In}$ this case, the cantilever initially behaves as having a negative damping constant $\left(Q_{\text {eff }}<0\right)$. Thus, during a brief period, the oscillation exponentially increases (rise time $\cong 2 Q_{\text {eff }} / \omega_{0}$ ) until the nonlinear saturation of the system comes into play and the oscillation achieves a steady state. The steady oscillation has small amplitude fluctuations due to the strong nonlinear potential imposed by the saturation and the tendency of the amplitude to grow due to the negative decay time of the transient. Thus, whereas the mechanical motion is equally distributed in amplitude and phase noises in the linear regime, the amplitude noise is largely squeezed and the phase undergoes a relatively slow diffusion process that leads to a stable oscillator frequency.

In Fig. 2(b), the noise of the resonance frequency shift is estimated by calculating its amplitude discrete Fourier transform (DFT) for a time span of $10 \mathrm{~min}$. The resonance frequency DFTs for the nondriven cantilever (Brownian motion) and FM-driven cantilever are also shown. The frequency stability for the FM driven cantilever ranges from

FIG. 2. (Color online) (a) Frequency spectra of the second flexural mode for a microcantilever in water excited by thermal forces (green line), frequency modulation method (blue line), and the self-excitation technique in the nonlinear regime (red line). (b) Amplitude DFT of the resonance frequency shift of the cantilever excited by thermal forces (green line), the frequency modulation technique (blue line), and the self-excitation technique (red line).

$2000 \mathrm{ppm}$ for a measurement bandwidth $B$ $=0.1 \mathrm{~Hz}-400 \mathrm{ppm}$ for $B=1 \mathrm{~Hz}$. The resonance frequency resolution obtained by the FM driving technique is comparable to the best values reported in liquids for nanomechanical sensors. ${ }^{15}$ However, the self-excitation technique leads to a dramatic improvement in the frequency resolution of about two orders of magnitude. The frequency stability goes from $10 \mathrm{ppm}$ for $B=0.1 \mathrm{~Hz}$ to $5 \mathrm{ppm}$ for $B=1 \mathrm{~Hz}$.

The capability of the self-excitation technique in the nonlinear regime is illustrated by measuring in real time the change of the fluid properties when $1 \%$ of glycerol was added to water. Figure 3 shows the experiment recording of the second mode resonance frequency shift for a bandwidth of $1 \mathrm{~Hz}$. The resonance frequency shift obtained by the FM driving technique at the same bandwidth is also plotted for comparison. The resonance frequency starts to decrease as glycerol diffuses inside the fluid cell until it reaches a shift of about $-80 \mathrm{~Hz}$. The resonance frequency then recovers its initial value in approximately $30 \mathrm{~min}$ after the total purging with water. A similar behavior can be discerned when the cantilever is driven by the FM signal, however, the noise is significantly higher.

The resonance frequency decrease implies an increase in the mass of the cantilever $\left(m_{c}\right)$ that in fluid can be split up into the mass in vacuum and the fluid mass dragged by the to AIP license or copyright; see http://apl.aip.org/apl/copyright.jsp 


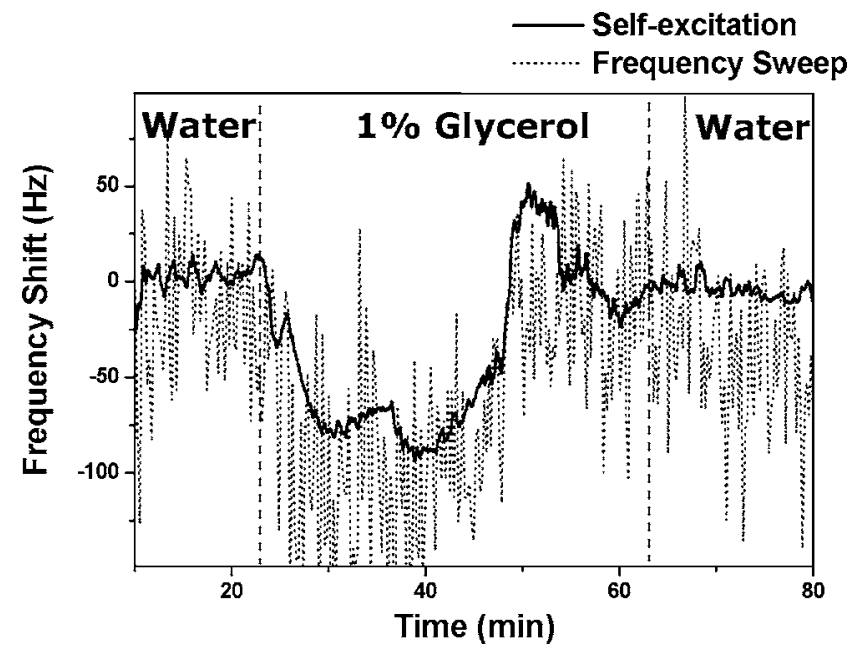

FIG. 3. Response of the resonance frequency of the second flexural mode of the microcantilever in water to the addition of $1 \%$ of glycerol $(\mathrm{m} / \mathrm{m})$. The resonance frequency shift was measured by the self-excitation technique (continuous line) and the frequency modulation method (dotted line) in two different experiments performed with the same experimental conditions.

cantilever. We estimate the mass of the cantilever by using the expressions $k \approx 0.24 m_{c} \omega_{1}^{2}$ and $\Delta \omega_{2} / \omega_{2} \approx-1 / 2 \Delta m_{c} / m_{c}$, where $\omega_{1}$ and $\omega_{2}$ are the angular resonance frequencies of the first and second flexural vibration modes of the cantilever, respectively. The cantilever mass in vacuum is of about $10.2 \mathrm{ng}$ and after immersion in water is approximately $211 \mathrm{ng}$. The addition of $1 \%$ of glycerol results in an increase in the dragged mass of about $0.8 \mathrm{ng}$. The value of the dragged mass is in consistency with the Navier-Stokes theory that predicts the following relationship between the mass of the cantilever and the fluid properties. ${ }^{16,17}$

$$
\frac{m_{c, f}}{m_{c, v}}=\left\{1+\frac{4 W \rho}{\rho_{c} T \pi} \operatorname{Re}[\Gamma(\omega)]\right\},
$$

where $m_{c, f}$ and $m_{c, v}$ are the cantilever mass in the fluid and in vacuum, $\rho$ is the fluid density, $\rho_{c}$ is the cantilever density, $W$ is the cantilever width, and $T$ is the cantilever thickness. $\Gamma(\omega)$ is the hydrodynamic function for the cantilever ${ }^{18}$ that depends on the Reynolds number $\operatorname{Re}=\rho \omega W^{2} / 4 \eta$, where $\eta$ is the viscosity of the fluid and $\omega$ is the vibration frequency. The theoretical calculation predicts a change of the dragged mass of $1.2 \mathrm{ng}$ by taking into account that the addition of $1 \%$ of glycerol in water gives an increase in the mass density and the viscosity of the fluid of $0.13 \%$ and $1.27 \%$, respectively.

The high frequency resolution demonstrated above arises from the singular dynamic behavior of self-sustained oscillators in the nonlinear regime. The frequency noise can be estimated by modeling the oscillation as one with zero amplitude noise and zero-mean stationary random phase, ${ }^{13}$

$$
\frac{\delta \omega}{\omega_{0}} \approx 0.114 \sqrt{\frac{k_{B} T k}{Q^{3} F_{s}^{2}} \frac{B}{\omega_{0}}},
$$

where $k_{B}$ is the Boltzmann's constant, $T$ is the absolute temperature, and $F_{s}$ is the maximum force provided by the actuator. Equation (2) predicts a frequency resolution of the order of $1 \mathrm{ppm}$ in consistency with the experiments shown here. This value allows, for instance, measuring mass adsorption with a sensitivity of $1 \mathrm{pg}$ that is an enhancement of three orders of magnitude with respect to state of the art. Major improvements can be obtained by using cantilevers with higher intrinsic quality factor and smaller spring constant. In addition, the sensitivity of nanomechanical sensors to mass adsorption (biosensors) or to dragged mass (rheological measurents) can be enhanced by decreasing the cantilever size. In fact cantilevers 100 times smaller have been used to measure mass adsorption at the attogram level in vacuum. ${ }^{5}$ The use of similar cantilevers in liquid would increase the sensitivity several orders of magnitude. In conclusion, the self-excitation technique described here together major improvements in the design of nanomechanical resonators open the door for unprecedented in situ exploration and detection of biological systems in real time.

D.R. acknowledges the fellowship funded by the Autonomic Community of Madrid. This research was supported by Spanish Ministry of Science under Grant No. TEC200610316 and Community of Madrid under Grant No. $200550 \mathrm{M} 056$.

${ }^{1}$ E. Meyer, H. J. Hug, and R. Bennewitz, Scanning Probe Microscopy: The Lab on a Tip (Springer, Berlin, 2003).

${ }^{2}$ P. S. Waggoner and H. G. Craighead, Lab Chip 7, 1238 (2007).

${ }^{3}$ F. R. Blom, S. Bouwstra, M. Elwenspoek, and J. H. J. Fluitman, J. Vac. Sci. Technol. B 10, 19 (1992).

${ }^{4}$ J. Tamayo, J. Appl. Phys. 97, 044903 (2005).

${ }^{5}$ K. L. Ekinci, X. M. H. Huang, and M. L. Roukes, Appl. Phys. Lett. 84, 4469 (2004).

${ }^{6}$ T. P. Burg, M. Godin, W. Shen, G. Carlson, J. S. Foster, K. Babcock, and S. R. Manalis, Nature (London) 446, 1066 (2007).

${ }^{7}$ D. M. Karabacak, V. Yakhot, and K. L. Ekinci, Phys. Rev. Lett. 98, 254505 (2007).

${ }^{8}$ J. A. Judge, J. F. Vignola, and J. Jarzynski, Appl. Phys. Lett. 92, 124102 (2008).

${ }^{9}$ S. Boskovic, J. W. Chon, P. Mulvaney, and J. E. Sader, J. Rheol. 46, 891 (2002).

${ }^{10}$ S. Basak, A. Raman, and S. V. Garimella, J. Appl. Phys. 99, 114906 (2006).

${ }^{11}$ D. Ramos, J. Tamayo, J. Mertens, and M. Calleja, J. Appl. Phys. 99, 124904 (2006).

${ }^{12}$ B. Ilic, S. Krylov, K. Aubin, R. Reichenbach, and H. G. Craighead, Appl. Phys. Lett. 86, 193114 (2005).

${ }^{13}$ J. Tamayo, M. Calleja, D. Ramos, and J. Mertens, Phys. Rev. B 76, 180201 (2007)

${ }^{14}$ T. Fukuma, M. J. Higgins, and S. P. Jarvis, Biophys. J. 92, 3603 (2007).

${ }^{15}$ T. Braun, V. Barwich, M. K. Ghatkesar, A. H. Bredekamp, C. Gerber, M. Hegner, and H. P. Lang, Phys. Rev. E 72, 031907 (2005).

${ }^{16}$ M. R. Paul, M. T. Clark, and M. C. Cross, Nanotechnology 17, 4502 (2006).

${ }^{17}$ M. Papi, G. Arcovito, M. De Spirito, M. Vassalli, and B. Tiribilli, Appl. Phys. Lett. 88, 194102 (2006).

${ }^{18}$ J. E. Sader, J. Appl. Phys. 84, 64 (1998). 